\title{
Mitochondrial genome sequencing reveals potential origins of the scabies mite Sarcoptes scabiei infesting two iconic Australian marsupials
}

Tamieka A. Fraser ${ }^{1,2}$, Renfu Shao ${ }^{2}$, Nicholas M. Fountain-Jones ${ }^{3}$, Michael Charleston ${ }^{1,4}$, Alynn Martin, Pam Whiteley ${ }^{5}$, Roz Holme ${ }^{6}$, Scott Carver ${ }^{1}$ and Adam Polkinghorne ${ }^{2^{*}}$ (D)

\begin{abstract}
Background: Debilitating skin infestations caused by the mite, Sarcoptes scabiei, have a profound impact on human and animal health globally. In Australia, this impact is evident across different segments of Australian society, with a growing recognition that it can contribute to rapid declines of native Australian marsupials. Cross-host transmission has been suggested to play a significant role in the epidemiology and origin of mite infestations in different species but a chronic lack of genetic resources has made further inferences difficult. To investigate the origins and molecular epidemiology of S. scabiei in Australian wildlife, we sequenced the mitochondrial genomes of S. scabiei from diseased wombats (Vombatus ursinus) and koalas (Phascolarctos cinereus) spanning New South Wales, Victoria and Tasmania, and compared them with the recently sequenced mitochondrial genome sequences of $S$. scabiei from humans.

Results: We found unique S. scabiei haplotypes among individual wombat and koala hosts with high sequence similarity (99.1\% - 100\%). Phylogenetic analysis of near full-length mitochondrial genomes revealed three clades of $S$. scabiei (one human and two marsupial), with no apparent geographic or host species pattern, suggestive of multiple introductions. The availability of additional mitochondrial gene sequences also enabled a re-evaluation of a range of putative molecular markers of $\mathrm{S}$. scabiei, revealing that cox 1 is the most informative gene for molecular epidemiological investigations. Utilising this gene target, we provide additional evidence to support cross-host transmission between different animal hosts.

Conclusions: Our results suggest a history of parasite invasion through colonisation of Australia from hosts across the globe and the potential for cross-host transmission being a common feature of the epidemiology of this neglected pathogen. If this is the case, comparable patterns may exist elsewhere in the 'New World'. This work provides a basis for expanded molecular studies into mange epidemiology in humans and animals in Australia and other geographic regions.
\end{abstract}

Keywords: Sarcoptes scabiei, Wombat, Koala, Mitochondrial genome sequencing, cox1, Phylogeny, Conservation

\section{Background}

Estimated as infecting 110 million humans and more than 100 mammalian species worldwide, Sarcoptes scabiei is widespread, burdensome, and has among the widest host range of known parasites [1, 2]. S. scabiei infests the epidermis of its hosts, causing a wide range of host

\footnotetext{
* Correspondence: apolking@usc.edu.au

${ }^{2}$ Centre for Animal Health Innovation, School of Science and Engineering,

University of the Sunshine Coast, Sippy Downs, QLD, Australia

Full list of author information is available at the end of the article
}

immune responses and associated mange disease symptoms (otherwise classified as scabies in humans) including skin irritation, inflammation, hyperkeratosis, alopecia, pruritis, rheumatic heart disease, and secondary bacterial infections [1]. The life cycle of this mite has five developmental stages (egg, larva, protonymph, tritonymph and adult) occurring within the stratum corneum, with durations of life cycle occurring between 7 and 21 days [3-5]. It is most commonly reported in humans, domestic dogs and livestock, with impacts to health, animal welfare and 
primary production $[4,6,7]$. Transmission of mites between individuals and hosts occurs by direct contact, sharing of contaminated materials or habitat with mites known to survive off its host for up to three weeks $[3,5]$. With a wide and expanding wildlife host range, this pathogen is also labelled an emerging infectious disease in North America and Australia [7].

Sarcoptic mange or scabies is a major parasitic disease of indigenous people and their domestic dogs, particularly in northern Australia [8-10]. In remote Aboriginal communities, up to $50 \%$ of children suffer from $S$. scabiei infestations, resulting in endemic transmission and severe cases of scabies induced pyoderma [9]. These communities also share communal space with mange infested dogs, a risk for continuous zoonotic transmission [10]. S. scabiei has also been documented to infest iconic Australian native species, including wombats (Vombatus ursinus, Lasiorhinus latifrons) [11], wallabies (Wallabie bicolor, Macropus agilis) [12, 13], koala (Phascolarctos cinereus) [14], southern brown bandicoot (Isoodon obesulus) [15], and dingo (Canis lupus dingo) [6]. Among these, wombats appear to experience the most severe pathology with mange associated with population declines exceeding $90 \%$ in some instances $[16,17]$. Recent studies suggests a rise in mange cases in koalas also, particularly in populations from southern Australia [18].

Despite large numbers of mange cases in these wildlife species, the origins and phylogenetic relationships of $S$. scabiei among host species remains significantly understudied. Only two studies have contributed to the genetic knowledge of Australian-derived mites and these have revealed contrasting results. The first of these studies utilized sequencing of partial S. scabiei $12 \mathrm{~S}$ rRNA gene sequence fragments [19], revealing close sequence similarity between mites from wombats, dogs and humans in Australia [19]. A subsequent investigation examining mites from Australian humans and animals and comparing them to mites from elsewhere, however, highlighted a strong relationship between S. scabiei mitochondrial DNA haplotypes based on host and geographic differences [10]. While no new sequences were obtained from Australian animals, most recently, a French study of $12 \mathrm{~S}$ rRNA and $\operatorname{cox} 1$ gene sequences from $S$. scabiei isolated from humans and dogs supported Skerratt et al.'s [19] original suggestion of a relationship between human, domestic animal and wombat sequences. It is, thus, proposed that occurrence of sarcoptic mange in wombats is the result of past spillover from humans and dogs, potentially from Europe. The exact relationship between mites infesting native Australian wildlife and the endemic strains shared between indigenous human populations and domesticated dogs is currently unknown due to the almost complete absence of any additional sequence data from these sources of S. scabiei [20].
Apart from the chronic shortage of genetic information for S. scabiei, considerable controversy exists over the choice of gene loci to be used for molecular typing of this pathogenic mite. The most common targets for S. scabiei typing studies to date have been the mitochondrial $12 \mathrm{~S}$ rRNA, 16S rRNA and Cytochrome oxidase 1 (cox1) genes. The $12 \mathrm{~S}$ rRNA gene is highly conserved and does not discriminate well between host species infected by $S$. scabiei, although some studies have used it for this purpose $[19,21]$. The $16 \mathrm{~S}$ rRNA gene is less conserved, though is also limited in its ability to identify genetic distinctions among S. scabiei mites separate hosts or locations [22, 23]. $\operatorname{cox} 1$ is the most variable of the three genes, with greater power to distinguish between mites from different host species [20, 24-26]. Nuclear markers have also been investigated including the use of microsatellites [27-30] and phylogenetic analysis of ITS-2 gene sequences $[23,25,31-34]$. The latter studies concluded that $S$. scabiei was a single heterogeneous species with a low level of genetic diversity [23, 25, 31-34]. Microsatellites studies have provided some genetic evidence for crossspecies infestation [29], with some limited ability to distinguish mites from different host groups but not by geographic location of isolation [27-30].

In an effort to gain insight into the origins of S. scabiei in Australian wildlife and to provide base-line data for studies to investigate the relationship to $S$. scabiei infestations of humans and domesticated animals, we sequenced the mitochondrial genomes of $S$. scabiei mites collected from bare-nosed wombats from Tasmania, New South Wales (NSW) and Victoria (VIC), and koalas from VIC. We used this new genetic information to assess the potential role of S. scabiei cross-host transmission to and from marsupial host species following detailed phylogenetic analysis. Because the choice of genetic loci for molecular epidemiology studies has influenced previous studies of $S$. scabiei origins and host specificity, we additionally examined individual mitochondrial genes to aid in the selection of gene targets for downstream investigations into the phylogenetic relationships of mites from a range of hosts and geographic locations, not only for Australian studies but for global comparisons.

\section{Methods}

\section{Mite collection and DNA extraction}

Mites were obtained via skin scrapings during necropsy from Victorian koalas $(N=5)$ and wombats from Victoria $(N=2)$ and Tasmania $(N=3)$ or as a part of routine veterinary care from anaesthetised New South Wales wombats $(\mathrm{N}=2)$. From each individual host, 1-3 mites were pooled for DNA extraction (to obtain sufficient mtDNA for sequencing) following protocol using a DNeasy Blood and Tissue kit (Qiagen). The term "mite" will be used here forth for pooled sequences of several individuals. 


\section{Mitochondrial DNA long range PCR}

To amplify full-length mitochondrial DNA sequences, primers were designed to cover four overlapping fragments (Additional file 1). Each long-range PCR was conducted on a thermocycler with a final PCR volume of $20 \mu \mathrm{L} ; 5 \mu \mathrm{L} 5 \mathrm{X}$ PrimeSTAR ${ }^{\circ}$ GXL DNA buffer (Takara), $2 \mu \mathrm{L}$ dNTP mixture $(2.5 \mathrm{mM}), 1 \mu \mathrm{L}$ PrimeSTAR ${ }^{\circ}$ GXL DNA Polymerase (Takara), $1 \mu \mathrm{L}$ of forward and reverse primers $(0.3 \mu \mathrm{M}), 9 \mu \mathrm{L}$ water and $1 \mu \mathrm{L}$ of DNA template. Cycling conditions consisted of 35 repeats of $98{ }^{\circ} \mathrm{C}$ for $10 \mathrm{~s}, 50{ }^{\circ} \mathrm{C}$ for $20 \mathrm{~s}$ and $68^{\circ} \mathrm{C}$ for $10 \mathrm{mins}$. The presence of amplicons of the expected size was visually verified on a $1 \%$ TBE agarose gel, prior to purification using QIAquick PCR purification kit (Qiagen). DNA sequences of purified amplicons were determined by paired-end sequencing on a HiSeq4000 (BGI, Hong Kong) at $100 \mathrm{Mb}$ coverage after passing quality control.

\section{S. scabiei mitochondrial genome assembly and annotation}

Read mapping was performed in Geneious 9.1.3 [35] using the Geneious mapper method, originally using the four primer pair sequences used for PCR amplification as the reference sequence at 5 iterations, $100 \%$ identity and a minimum overlap of the same length of the primers ( $\operatorname{cox} 1$ at $22 \mathrm{bp}, 12 \mathrm{~S}$ rRNA at $30 \mathrm{bp}, c o b$ at $26 \mathrm{bp}$ and $n d 4$ at $32 \mathrm{bp}$ ). Reads were then mapped to the four output contigs at 100 bp overlap, 99\% identity and 100 iterations. Contigs produced from this assembly were aligned using MUSCLE alignment [36] in Geneious version 9.1.3 [35]. Assembly of the new mitochondrial genome sequences obtained in this study was confirmed by sequence alignment against the previously published human S. scabiei mitochondrial genome (accession number LN874268).

Mitochondrial protein coding genes were annotated by identifying open reading frames (ORF) in Geneious and blastp to confirm ORF identification and protein length. tRNA genes were identified using ARWEN [37]. The two rRNA genes were identified by blastn search of GenBank [38]. DnaSP [39] was used to assess the magnitude selective pressure by $\mathrm{dN} / \mathrm{dS}$ rations for all protein coding genes.

\section{Phylogenetic tree analysis}

The relationship between the new S. scabiei mitochondrial genomes and the available human derived mite was conducted by neighbour-net analysis at 1000 bootstrap replicates using SplitsTree (version 4.14.4) [40], showing only bootstrap values greater than 80 . Individual full length gene tree outputs of $16 \mathrm{~S}$ rRNA, cox1, cox3, cytb and ND4 were constructed with Mr. Bayes [41] in Geneious version 9.1.3, and were then compared to the Mr. Bayes tree output produced by the near full length (NFL) mitochondrial genome. Node tips and clade outputs were then visually compared between the two trees of interest, with associated tip lines drawn between the trees for clarity.

Global cox 1 phylogenetic analysis was completed using the current 12 mitochondrial genomes and the 78 available cox1 sequences in GenBank. All cox1 sequences were unified by trimming to $387 \mathrm{bp}$ to allow alignment of sequences of the same length. Phylogenetic tree construction on the resulting 90 sequences was performed by Mr. Bayes and rooted with Otodectes cynotis mitochondrial genome (KP676688). Bootstrap values greater than 80 were shown.

\section{Results}

S. scabiei mitochondrial genome assembly and annotation identify new haplotypes in Australian marsupials

Successful full-length mitochondrial genome assembly was confirmed for all samples (accession numbers MF083732MF083743). Neither single nor pooled DNA extraction played a significant role in amplification or exhibited sequencing errors. The mitochondrial genome size ranged between 13,830 bp and 13,926 bp with variations in length largely owing to the non-coding repeat region of "ATs". The mitochondrial genome GC content was comparable amongst all samples, and all new S. scabiei from koala and wombats were $>98 \%$ similar to the mite genome from humans (Table 1).

Thirteen protein coding genes were identified in all 12 mitochondrial genomes using the invertebrate mitochondrial genetic code. tRNA annotation (ARWEN) resolved the presence of 16 of the 22 tRNA genes, with a further four added $(\operatorname{trn} Q, \operatorname{trn} R, \operatorname{trn} P$ and $\operatorname{trn} F)$ based on a visual inspection of the mitochondrial genome sequences and sequence identity to the previously annotated human $S$. scabiei mitochondrial genome (LN874268) [42]. Two invertebrate tRNA genes ( $\operatorname{trn} A$ and $\operatorname{trn} Y$ ) were not identified, however these were also missing in the human S. scabiei mitochondrial genome (LN874268) [42].

Sequence analysis of the wombat and koala mite mitochondrial genomes, after removing the repeat region variation between $\operatorname{trnS} 1$ and $\operatorname{trnF}$, revealed the presence of 11 unique haplotypes that shared between $99.1 \%$ and $100 \%$ sequence identity against the human $S$. scabiei mitochondrial genome. All NFL mitochondrial genomes were the same length when aligned (13,822 bp). Between the new genomes, only two koala mites (K4 and K7) shared 100\% sequence similarity. The highest number of differences occurred between two wombat mites, N11 and DW02, from NSW and Tasmania respectively (171 nucleotides). The NFL mitochondrial genome of the human S. scabiei mite had the highest number of differences to a NSW wombat mite (N11) (178 nucleotide) and the lowest number of differences to a Victorian koala (K8) (83 nucleotides). 
Table 1 Details of new mitochondrial genomes, size, GC content and their sequence similarity to the human S. scabiei mite (LN874268)

\begin{tabular}{|c|c|c|c|c|c|}
\hline Sample Name & Host (Location) & Pooled/Single Mite & Genome Size (bp) & GC \% & \% Similarity to LN874268 \\
\hline DW02 & Wombat (Narawntapu, Tasmania) & Single & 13,911 & 19.21 & 99.44 \\
\hline S1 & Wombat (South Swansea, Tasmania) & Single & 13,839 & 19.37 & 99.03 \\
\hline B1 & Wombat (Brighton, Tasmania) & Single & 13,887 & 19.23 & 99.47 \\
\hline N11 & Wombat (Central Coast, New South Wales) & Pooled & 13,845 & 19.39 & 98.62 \\
\hline N22 & Wombat (Murrys Run, New South Wales) & Single & 13,900 & 19.32 & 98.98 \\
\hline W2 & Wombat (Kangaroo Ground, Victoria) & Pooled & 13,856 & 19.33 & 99.06 \\
\hline W & Wombat (Arthurs Creek, Victoria) & Pooled & 13,848 & 19.35 & 99.16 \\
\hline K2 & Koala (Koonoomoo, Victoria) & Pooled & 13,926 & 19.29 & 99.43 \\
\hline K4 & Koala (Koonoomoo, Victoria) & Pooled & 13,828 & 19.39 & 99.18 \\
\hline K5 & Koala (Sandy Point, Victoria) & Pooled & 13,830 & 19.40 & 99.21 \\
\hline K7 & Koala (Koonoomoo, Victoria) & Pooled & 13,854 & 19.35 & 98.86 \\
\hline K8 & Koala (Koonoomoo, Victoria) & Pooled & 13,846 & 19.37 & 99.39 \\
\hline
\end{tabular}

Phylogenetic analysis reveals a range of S. scabiei haplotypes among koalas and wombats

SplitsTree phylogenetic analysis of complete S. scabiei near full-length mitochondrial genomes revealed three major clades (bootstrap support $>0.8$ ), (Fig. 1); one is exclusively comprised of the human mite (LN874268) and the remaining two are a mix of marsupial mites. Each marsupial lineage could be further subdivided into two smaller clades comprising: (i) wombat mites from Tasmania (DW02 and Brighton), (ii) wombat (WV) and koala mites (K8 and K2) from Victoria, (iii) wombat mites from New South Wales (N11 and N22) and Victoria (W2), and; (iv) mites from a single wombat from Tasmania (S1) and koalas from Victoria (K4, K5 and K7). With the exception of subclade iii, all subclades are strongly supported by the length of parallel splits and their corresponding bootstraps. No clear host or geographical pattern was evident between the two major marsupial mite clades. We conducted a range of phylogenetic analyses all emitting the same result (Additional file 2).

\section{Co-phylogenetic comparisons show individual mitochondrial genes vary in their utility for molecular epidemiological investigations}

The expanded set of mitochondrial genome sequences for S. scabiei generated in this study enabled an evaluation of several mitochondrial genetic markers for molecular epidemiology studies. DnaSP [43] analysis revealed that (i) every mitochondrial gene is under negative selection with atp 8 to be the closest to neutral selection, (ii) $n d 4$ had the highest number of parsimony informative sites and, (ii) $c y t b$ identified the largest number of different haplotypes (Table 2).

Using the five genes with the greatest number of unique haplotypes (16S rRNA 8, $\operatorname{cox} 19, \operatorname{cox} 38, c y t b 10$ and $n d 48$ ), a co-phylogenetic comparison of their trees was made to the NFL phylogeny (Fig. 2). All trees were rooted with the human mite (LN874269) to allow comparisons of clade construction and analysis of how the new haplotypes relate to each other. The phylogenetic trees constructed from each of the five genes portrayed the three major clades shown by analysis of the NFL mitochondrial genomes. Noticeably, the phylogenetic trees constructed on two of these genes $(\operatorname{cox} 1$ and $n d 4)$ also revealed the same subclade structure. For cox3 and $16 \mathrm{~S}$ rRNA genes, the low number of haplotypes identified revealed less resolution of putative ancestral linages. $C y t b$ and $16 \mathrm{~s}$ rRNA genes differed with the position of $\mathrm{K} 5$ and W2 alternating between with the two NSW wombat mite clades and the VIC koala and Tasmanian wombat mite clade. The change in relative position of $\mathrm{K} 5$ and W2 in the latter trees expands a subclade that is predicted to include only wombat sequences based on NFL mitochondrial sequence analysis to also include sequences from koalas.

cox1 analysis reveals that Australian marsupials share $S$. scabiei haplotypes with a diverse range of globally distributed mites

We combined our new $\operatorname{cox} 1$ sequences with other global cox 1 haplotypes from across the globe [20, 24, 44] to perform a phylogenetic analysis to elucidate the relationships between Australian mites and those from animals and humans elsewhere. Sequence comparison and clade outgroups identified five haplotypes: (i) K7, S1, K4 and K5 were identical to a dog mite and three racoon dog mites from Japan (AB821011, AB821004, A821007, AB821012), (ii) W2 was identical to a dog mite from China (KT961022), a dog mite from Italy (KT961025), a single dog mite and three fox mites from France (KT961029, KT961030, KT961031, KT961032), a dog mite from the USA (AY493393) and a wombat mite and wallaby mite from Australia (AY493397 and AY493398), 


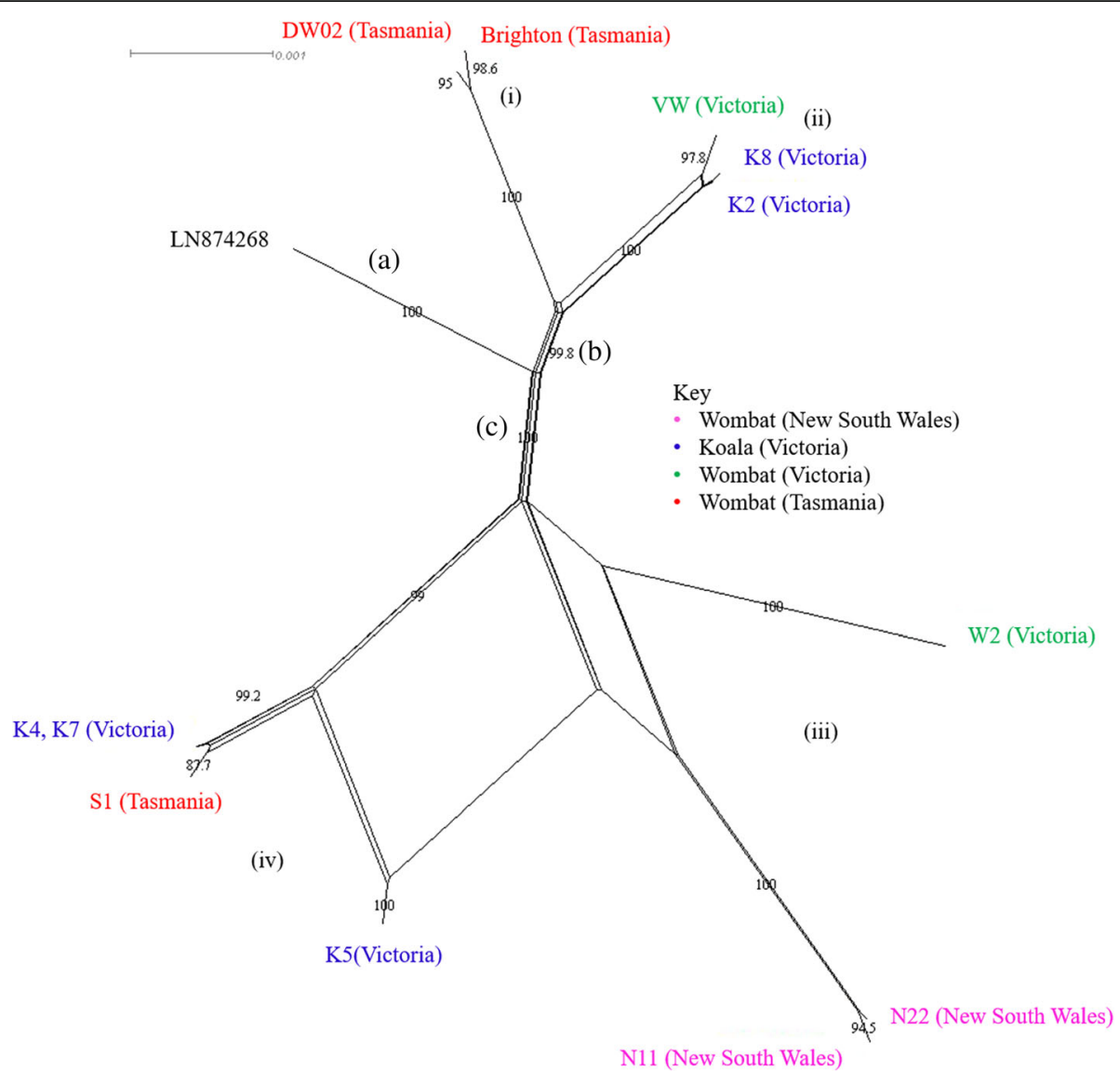

Fig. 1 SplitsTree anlaysis of new near full-length mitochondrial genomes for koala and wombat S. scabiei. Three major clades are identifed by splits: a the human mite LN874269, b marsupial mites from Tasmania and Victoira and, c marsupial mites from New South Wales, Victoria and Tasmania. Four subclades are further recognized, labeled (i-iv)

Table 2 Comparison of S. scabiei mitochondrial genes from koala and wombat hosts showing the number of informative sites, haplotypes and the magnitude of natural selection (dN/dS)

\begin{tabular}{lllll}
\hline Gene locus & Length (bp) & $\begin{array}{l}\text { Number of parsimony } \\
\text { informative sites }\end{array}$ & $\mathrm{dN} / \mathrm{dS}$ & $\begin{array}{l}\text { Number of } \\
\text { haplotypes }\end{array}$ \\
\hline 12S rRNA & 657 & 4 & NA & 7 \\
16S rRNA & 1040 & 13 & NA & 8 \\
atp8 & 159 & 4 & 0.705 & 4 \\
cox1 & 1551 & 17 & 0.040 & 9 \\
cox2 & 751 & 9 & 0.031 & 6 \\
cox3 & 783 & 13 & 0.022 & 8 \\
Cytb & 1101 & 16 & 0.066 & 10 \\
nd1 & 900 & 11 & 0.055 & 6 \\
nd2 & 928 & 12 & 0.245 & 6 \\
nd3 & 354 & 3 & 0.105 & 7 \\
nd41 & 255 & 3 & 0.000 & 3 \\
nd4 & 1297 & 21 & 0.132 & 8 \\
nd5 & 1626 & 20 & 0.040 & 7 \\
nd6 & 441 & 4 & 0.000 & 5 \\
\hline
\end{tabular}

(iii) B1 and DW02 were the same and unique to everything else, (iv) VW, K2 and K8 are identical to a single dog mite from Australia (AY493391) and, (v) N11 and N22 were identical to a jackal mite, a buffalo mite and two sheep mites from Egypt (KP987792, AB779594, AB779608, AB779609). From the phylogenetic tree (Fig. 3) haplotypes containing the Tasmanian wombats (B1 and DW02) and Vic marsupials (VW, K2 and K8) are the only sequences that sit within other human sequences from Australia and France and dog sequences from China. All other human sequences are within its own distinct major clade.

\section{Discussion}

Pathogen dispersal and spillover likely underscore global infestations of S. scabiei among many animal species, including Australian wildlife [45]. However, a dearth of genetic information for this mite has hindered molecular epidemiological investigations. We tackled this problem with mitochondrial genome sequencing of $S$. scabiei across a broad geographic range of two impacted marsupial host species. The comparison of NFL 


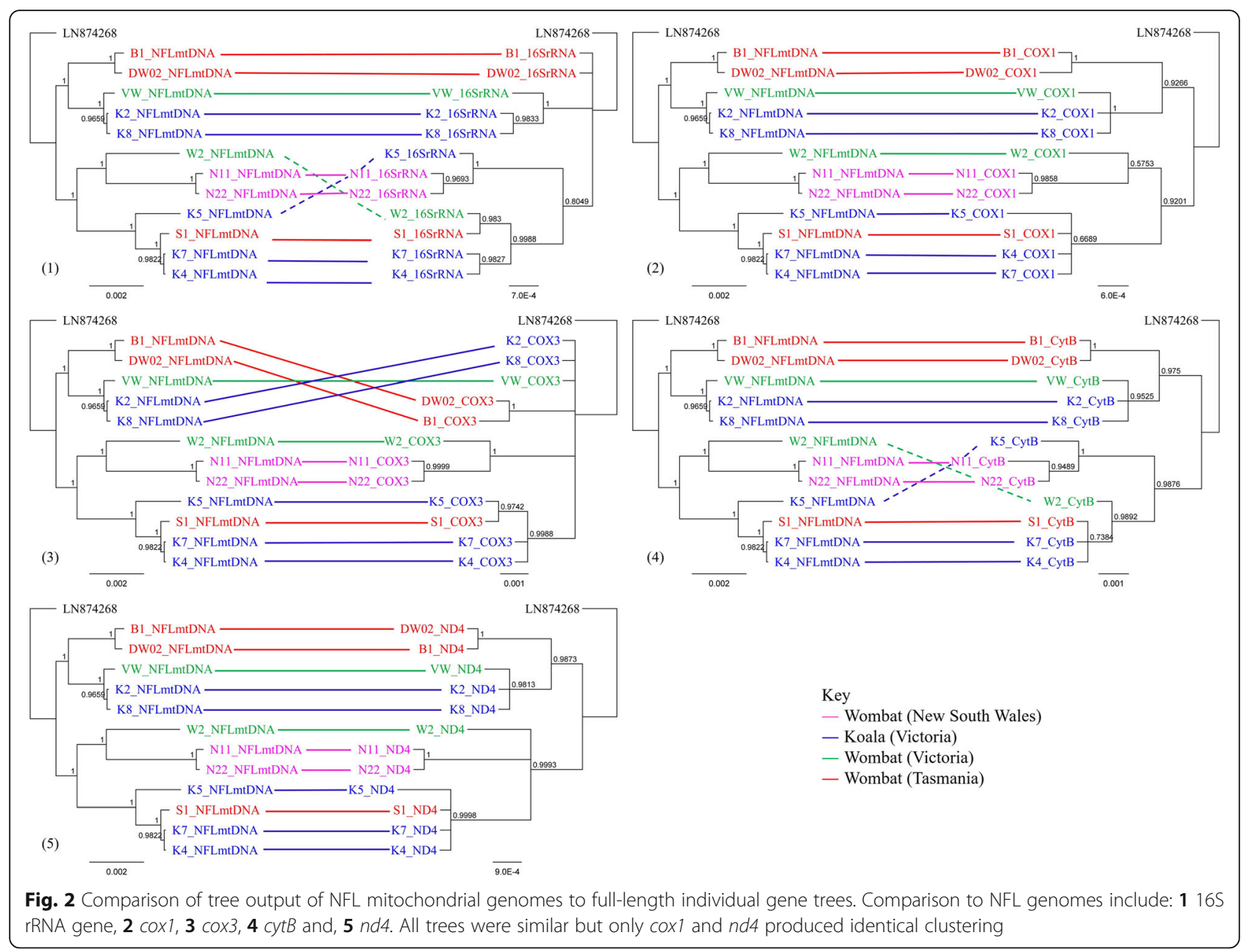

genome assemblies showed unique haplotypes infesting koalas and wombats across south-eastern Australia. Despite high NFL sequence similarity (>99\%), our analysis revealed 11 unique mitochondrial haplotypes, with only two koala mites sharing $100 \%$ sequence identity.

The presence of at least two major evolutionary lineages of S. scabiei occurring among wombats in Tasmania, and wombats and koala mites from Victoria suggests a polyphyletic origin may exist for S. scabiei in Australia. The absence of major clade separation between wombats and koala mites suggests a level of pathogen generality, indicative of multiple cross-species transmission events. Although we did not sample other animals in this study, we think it is reasonable to hypothesise that these crossspecies transmission events may also include other animals such as domestic and wild dogs and invasive foxes.

Although more sampling is clearly required to address this question conclusively, our observations supports the hypothesis that S. scabiei was first introduced to Australian wildlife in the time following European colonisation. This is based on (i) significant sequence similarity in the NFL mitochondrial genomes of the marsupial mites sampled in this study and; (ii) the single gene molecular typing results that reveal that cox 1 haplotypes are shared between marsupial mites and those from a diverse range of animal hosts in the rest of the world, Indeed this is not a new suggestion [11, 19-21], but our data provides the strongest support yet for this hypothesis. Historical records of mange disease in humans and domestic dogs from Tasmania date back to as early as the 1820s [46, 47], suggesting that Australian wildlife have been infested by $S$. scabiei for at least 200 years. The main question appears to simply be how long before then were mites introduced. Pre-European hypotheses must still be considered (albeit weakly) plausible, including introduction through aboriginal colonisation (ca. 50,000-60,000 years ago), introduction of the dingo (ca 4000 years ago), or deeper evolutionary origins. If $S$. scabiei mitochondrial genome evolutionary rates are similar to that of other invertebrates (i.e. $10^{-6}$ substitutions per year $[48,49])$, then the evolutionary timescale of Australian marsupial mite divergence would be likely greater than $>200$ years. This prediction, however, does not take into account potential changes to this rate associated with spillover into a new host and the obvious limitations 


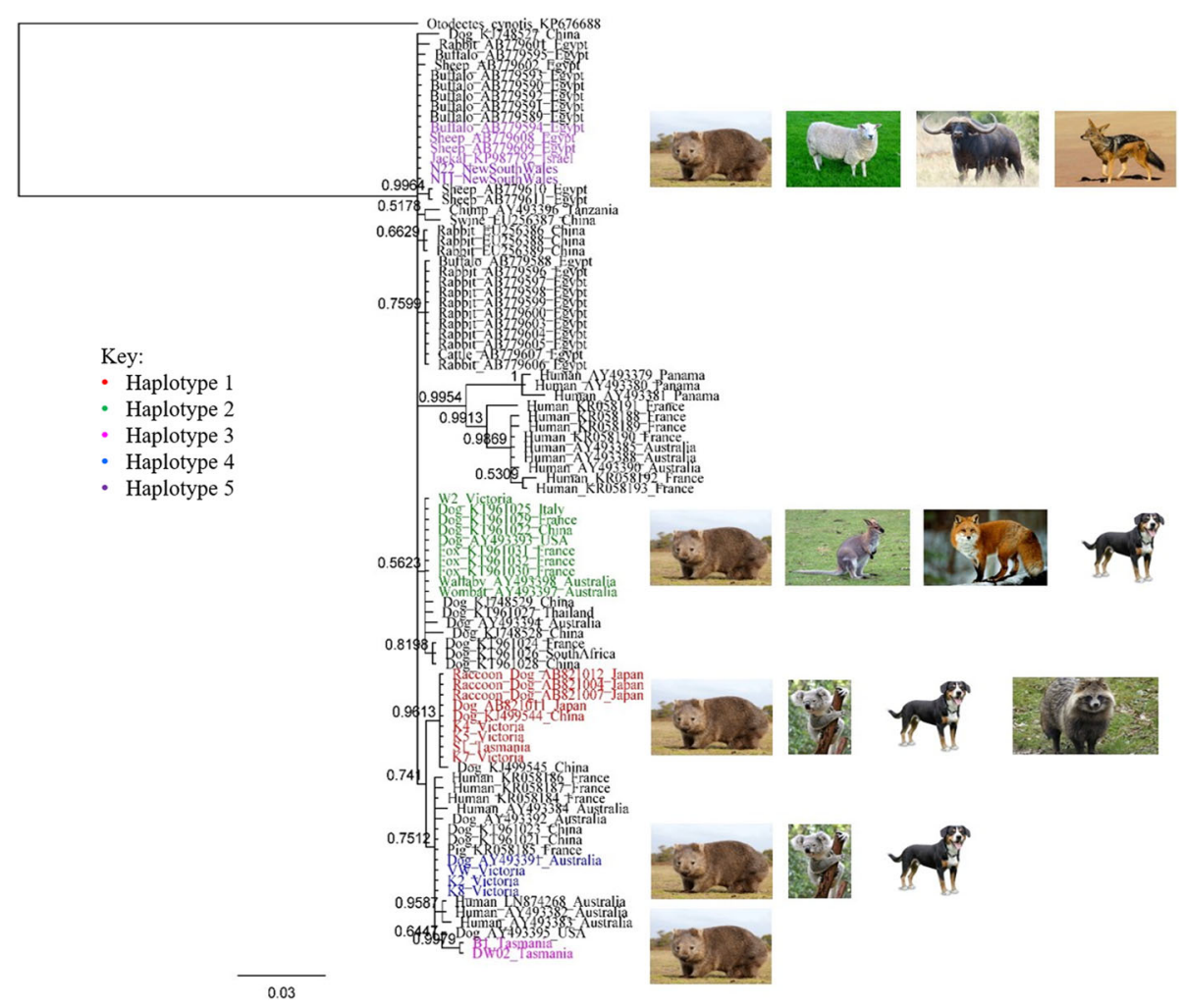

Fig. 3 Phylogenetic analysis of global cox 1 sequences. Comparison of 78 available cox 1 sequences in GenBank were used to construct a global phylogenetic tree, including the current Australian mitochondrial genomes, with Otodectes cynotis mitochondrial genome (KP676688) as the outgroup. Sequence comparison and clade outgroups identified five haplotypes which the new marsupial mite sequences were identical to as shown by colour and images of hosts for each haplotype. Each sequence from GenBank is labels as Host_Accession Number_Location

of making these interpretations on such a small sample set. Although a less parsimonious explanation, we also cannot obviously exclude the potential for mitochondrial capture and/or selective sweep phenomena to potentially mask the genetic signature of mites that may have been present in Australian animals prior to European colonisation. The resolution of the origins of $S$. scabiei in Australia will require sampling of mites from a greater time span from humans, marsupials and other hosts or a significant expansion in genomic sequence data for S. scabiei.

The availability of an extended set of mitochondrial sequences beyond the first initial human S. scabiei mitochondrial genome sequence provided an opportunity to re-assess the suitability of different mitochondrial genetic markers for detailed molecular epidemiology. Our research confirms that the two mitochondrial rRNA genes (12S and 16S) which have been used to infer origins of S. scabiei to Australia in the past, provide insufficient resolution for such assessments. The 16S rRNA gene, which is the more variable of the two genes (eight haplotypes identified in this study), can differentiate mites from differing host families, but cannot separate host species and location specific S. scabiei [20, 22], or preserve the resolution of ancestral origin when compared to the NFL mitochondrial genome. It has been suggested that cox 1 should be used as mitochondrial genetic marker due to the high variability and its ability to distinguish between biogeographical regions, host families, genera, species and even subspecies [20, 22, 50, 51]. Here, we also support this suggestion, with the phylogenetic reconstruction of the gene preserving an identical tree to the NFL mitochondrial genome. In the absence of available NFL genetic data for downstream molecular epidemiological investigations of S. scabiei in humans and animals, our analysis would appear to support the continued use of $\operatorname{cox} 1$ as a gene target for molecular typing.

To explore this further, we combined the new $\operatorname{cox} 1$ sequences from this study with new haplotypes from Europe [24] and other global haplotypes [20] to interrogate phylogenetic associations among hosts globally. Confirming our previous research [20], we found the Australian $S$. scabiei haplotypes share sequence identity with mites from non-human hosts in Europe, the Middle-East and Asia. These multiple geographic associations among a variety of host species potentially supporting multiple introduction events of S. scabiei from differing geographic regions into Australia, as indicated by the two mitochondrial genome clades shown in this study and the presence of multiple 
genetically distinct mites in Tasmania, some of which are also found on the mainland. Cross-host transmission has previously been demonstrated in the case of wombat to human transmission [52] and other experimental crossinfestations [31, 45, 53]. While clear genetic evidence for cross-host transmission between Australian marsupials and other hosts is lacking at this stage, the observation of shared cox 1 gene sequences (and similar NFL mitochondrial genome sequence data from this study) between Australian marsupial species suggests this is very likely. This observation alone highlights the need for additional precautions in the management of mange between different Australian wildlife and a renewed emphasis on infection control practices for stakeholders involved in their care.

\section{Conclusions}

This study is the first to sequence the mitochondrial genomes of S. scabiei in Australian marsupials. In doing so, we revealed a high level of sequence similarity among the marsupial mite sequences supporting the likely transmission of these mites between marsupial hosts affected. A greater repertoire of mites from more marsupial hosts spanning across Australia and other hosts globally would be beneficial. Mites from dingos, foxes and wild dogs in Australia would be particularly ideal as they likely contribute to the intra- and inter-specific transmission dynamics of S. scabiei on mainland Australia. Furthermore, an expansion of sample collection over time and geographical areas would also be beneficial for estimating rates of evolution, allowing evolutionary divergence to be better assessed. Our comparison of gene trees confirmed the use of $\operatorname{cox} 1$ as the most informative gene (when sequenced in full length) for phylogenetic comparisons. An expansion of such studies leveraging the genetic data provided in this study is expected to provide further insight into the global dissemination of this widespread and neglected human and animal pathogen.

\section{Additional files}

Additional file 1: Primer details for long range PCR. The four long range PCR fragments spaning across the mitochondrial genome are $4.4 \mathrm{~kb}, 4.0 \mathrm{~kb}$, $3.8 \mathrm{~kb}$ and $1.7 \mathrm{~kb}$ long. These primers are positioned on four main genes; 125 rRNA gene, $n d 4$, cob and cox1. (DOCX $13 \mathrm{~kb}$ )

Additional file 2: Additional phylogenetic trees constructed for new NFL mitochondrial genomes. Five different phylogenetic trees were constructed; 1) Log-Det NeightbourNet, 2) Raw (Uncorrected) proportional distance and Neighbour-Net, 3) Tree-inference method with Jukes-Cantor (1969) distances, 4) Neighbour-joining tree using uncorrected distances and 5) Neighbour-joining tree with bootstrap values. (DOCX $343 \mathrm{~kb}$ )

\section{Abbreviations}

NFL: Near full length; NSW: New South Wales; ORF: Open reading frame; PCR: Polymerase chain reaction; rRNA: ribosomal RNA; S. scabiei: Sarcoptes scabiei; tRNA: transfer RNA; VIC: Victoria

\section{Acknowledgments}

We would like to thank colleagues in the Faculty of Veterinary and Agricultural Sciences, The University of Melbourne, Wildlife Carers, Veterinary Practitioners, Parks Victoria and DELWP staff and Wildlife Health Australia, The Youngman Trust, The Vizard Foundation, and donors who contribute to Wildlife Health Surveillance Victoria and Kevin Holme at the Cedar Creek Wombat Hospital. We would also like to thank Martina Jelocnik and Alyce Taylor-Brown for their help in genome assembly and submission, and Keeley Thomas for her helping hand in the lab.

\section{Funding}

This work was funded by the Holsworth Wildlife Research Endowment to TF, SC and AP, and the Cradle Coast NRM to SC. The funders had no role in study design, data collection and analysis, decision to publish, or preparation of the manuscript.

\section{Availability of data and materials}

Mitochondrial genomes generated during the current study are available in GenBank repository with the accession numbers MF083732-MF083743.

\section{Authors' contributions}

Conceived and designed experiments: TF, AP, SC, and RS. Collected samples: PM, AM, RH and TF. Phylogenetic analysis: TF, NFJ, RS and MC. Wrote the paper: TF, NFJ, RS, MC, RH, AM, PM, SC and AP. All authors read and approved the final manuscript.

\section{Ethics approval}

This study and the collection of wombat and koala samples was approved by the Animal Research Committee at the University of the Sunshine Coast (approval AN/S/16/43) and the Animal Research Committee at the University of Tasmania (approval A0014670). Scientific purposes for the sampling of wildlife were provided by the relevant state departments including the Department of Primary Industries, Park, Water and Environment for Tasmania (approval FA15121), Office of Environment \& Heritage NSW National Parks \& wildlife Service (approval SL101719) and the Victorian Department of Environment and Primary Industry Research (approval 10,006,948, 10,008,033). All methods were carried out in accordance with the 2013 Australian National Health and Medical Research Council 'Australian code for the care and use of animals for scientific purposes'.

\section{Consent for publication}

Not Applicable.

Competing interests

The authors declare that they have no competing interests.

\section{Publisher's Note}

Springer Nature remains neutral with regard to jurisdictional claims in published maps and institutional affiliations.

\section{Author details}

${ }^{1}$ School of Biological Sciences, University of Tasmania, Sandy Bay, Hobart, TAS, Australia. ${ }^{2}$ Centre for Animal Health Innovation, School of Science and Engineering, University of the Sunshine Coast, Sippy Downs, QLD, Australia. ${ }^{3}$ University of Minnesota, Minneapolis, MN, USA. ${ }^{4}$ School of Information Technologies, University of Sydney, Camperdown, NSW, Australia. ${ }^{5}$ Faculty of Veterinary and Agricultural Sciences, The University of Melbourne, Werribee, VIC, Australia. ${ }^{6}$ Cedar Creek Wombat Rescue Inc. \& Hospital, Cedar Creek, NSW, Australia.

Received: 23 June 2017 Accepted: 21 November 2017 Published online: 28 November 2017

\section{References}

1. Pence D, Ueckermann E. Sarcoptic manage in wildlife. Rev Sci Tech. 2002;21(2):385-98.

2. Vos T, Flaxman AD, Naghavi M, Lozano R, Michaud C, Ezzati M, Shibuya K, Salomon JA, Abdalla S, Aboyans V. Years lived with disability (YLDs) for 1160 sequelae of 289 diseases and injuries 1990-2010: a systematic analysis for the global burden of disease study 2010. Lancet. 2013;380(9859):2163-96. 
3. Wendel K, Rompalo A. Scabies and pediculosis pubis: an update of treatment regimens and general review. Clinical Infect Dis. 2002; 35(Supplement_2):S146-51.

4. Walton SF, Holt DC, Currie BJ, Kemp DJ. Scabies: new future for a neglected disease. Adv Parasitol. 2004;57:309-76.

5. Arlian LG, Morgan MS. A review of Sarcoptes scabiei: past, present and future. Parasit Vectors. 2017;10(1):297.

6. McCarthy J, Kemp DJ, Walton SF, Currie BJ. Scabies: more than just an irritation. Postgrad Med J. 2004;80(945):382-7.

7. Tompkins DM, Carver S, Jones ME, Krkošek M, Skerratt LF. Emerging infectious diseases of wildlife: a critical perspective. Trends Parasitol. 2015;31(4):149-59.

8. Munoz E, Powers JR, Nienhuys TG, Mathews JD. Social and environmental factors in 10 aboriginal communities in the northern territory: relationship to hospital admissions of children. Med J Aust. 1992;156(8):529-33.

9. Currie BJ, Carapetis JR. Skin infections and infestations in aboriginal communities in northern Australia. Australas J Dermatol. 2000;41(3):139-43.

10. Walton S, Dougall A, Pizzutto S, Holt D, Taplin D, Arlian L, Morgan M, Currie B, Kemp D. Genetic epidemiology of Sarcoptes scabiei (Acari: Sarcoptidae) in northern Australia. Int J Parasitol. 2004;34(7):839-49.

11. Skerratt L, Martin R, Handasyde K. Sarcoptic mange in wombats. Aust Vet J. 1998;76(6):408-10

12. Holz PH, Orbell GMB, Beveridge I. Sarcoptic mange in a wild swamp wallaby (Wallabia bicolor). Aust Vet J. 2011;89(11):458-9.

13. McLelland D, Youl J. Sarcoptic mange in agile wallabies (Macropus agilis) in the northern territory. Aust Vet J. 2005;83(12):744-5.

14. Obendorf DL. Causes of mortality and morbidity of wild koalas, Phascolarctos cinereus (Goldfuss), in Victoria, Australia. J Wildl Dis. 1983;19(2):123-31.

15. Wicks R, Clark P, Hobbs R. Clinical dermatitis in a southern brown bandicoot (Isoodon obesulus) associated with the mite Sarcoptes scabiei. Comp Clin Path. 2007;16(4):271-4

16. Simpson K, Johnson CN, Carver S. Sarcoptes scabiei: the mange mite with mighty effects on the common wombat (Vombatus ursinus). PLoS One. 2016;11(3):e0149749.

17. Gray D. Sarcoptic mange affecting wild fauna in new South Wales. Aust Vet J. 1937;13(4):154-5.

18. KN Speight PW, L Woolford, PJ Duignan, B Bacci, S Lathe, WSJ Boardman, TF Scheelings, O Funnell, G Underwood, MA Stevenson. : Outbreaks of sarcoptic mange in free-ranging koala populations in Victoria and South Australia: a case series. Aust Vet J Accepted for publication 26/04/2017, 2017;95(7):244-249.

19. Skerratt L, Campbell N, Murrell A, Walton S, Kemp D, Barker S. The mitochondrial $12 \mathrm{~S}$ gene is a suitable marker of populations of Sarcoptes scabiei from wombats, dogs and humans in Australia. Parasitol Res. 2002;88(4):376-9.

20. Fraser TA, Charleston M, Martin A, Polkinghorne A, Carver S. The emergence of sarcoptic mange in Australian wildlife: an unresolved debate. Parasit Vectors. 2016;9(1):1-11.

21. Andriantsoanirina V, Ariey F, Izri A, Bernigaud C, Fang F, Guillot J, Chosidow O, Durand R. Wombats acquired scabies from humans and/or dogs from outside Australia. Parasitol Res. 2015;114(6):2079-83.

22. Mandal SD, Chhakchhuak L, Gurusubramanian G, Kumar NS. Mitochondrial markers for identification and phylogenetic studies in insects-A Review. DNA Barcodes. 2014:2(1):1-9.

23. Makouloutou P, Suzuki K, Yokoyama M, Takeuchi M, Yanagida T, Sato H. Involvement of two genetic lineages of Sarcoptes scabiei mites in a local mange epizootic of wild mammals in Japan. J Wildl Dis. 2015;51(1):69-78.

24. Andriantsoanirina $V$, Fang F, Ariey F, Izri A, Foulet F, Botterel F, Bernigaud C, Chosidow O, Huang W, Guillot J. Are humans the initial source of canine mange? Parasit Vectors. 2016;9(1):177.

25. Amer S, El Wahab TA, Metwaly AEN, Ye J, Roellig D, Feng Y, Xiao L. Preliminary molecular characterizations of Sarcoptes scaibiei (Acari: Sarcoptidae) from farm animals in Egypt. PLoS One. 2014;9(4):e94705.

26. Zhao Y, Cao Z, Cheng J, Hu L, Ma J, Yang Y, Wang X, Zeng J, Wang T. Population identification of Sarcoptes hominis and Sarcoptes canis in China using DNA sequences. Parasitol Res. 2014:1-10.

27. Alasaad S, Oleaga Á, Casais R, Rossi L, Molinar-Min A, Soriguer RC, Gortázar C. Temporal stability in the genetic structure of Sarcoptes scabiei under the host-taxon law: empirical evidences from wildlife-derived Sarcoptes mite in Asturias, Spain. Parasit Vectors. 2011;4:151.

28. Gakuya F, Rossi L, Ombui J, Maingi N, Muchemi G, Ogara W, Soriguer RC, Alasaad S. The curse of the prey: Sarcoptes mite molecular analysis reveals potential prey-to-predator parasitic infestation in wild animals from Masai Mara, Kenya. Parasit Vectors. 2011:4:193.
29. Rasero R, Rossi L, Soglia D, Maione S, Sacchi P, Rambozzi L, Sartore S, Soriguer RC, Spalenza V, Alasaad S. Host taxon-derived Sarcoptes mite in European wild animals revealed by microsatellite markers. Biol Conserv. 2010;143(5):1269-77.

30. Soglia D, Rasero R, Rossi L, Sartore S, Sacchi P, Maione S. Microsatellites as markers for comparison among different populations of Sarcoptes scabiei. Ital J Anim Sci. 2007;6(1s):214-6.

31. Alasaad S, Soglia D, Spalenza V, Maione S, Soriguer RC, Perez JM, Rasero R, Degiorgis MP, Nimmervoll H, Zhu XQ, et al. Is ITS-2 rDNA suitable marker for genetic characterization of Sarcoptes mites from different wild animals in different geographic areas? Vet Parasitol. 2009;159(2):181-5.

32. Berrilli F, D'Amelio S, Rossi L. Ribosomal and mitochondrial DNA sequence variation in Sarcoptes mites from different hosts and geographical regions. Parasitol Res. 2002;88(8):772-7.

33. Gu X-B, Yang G-Y. A study on the genetic relationship of mites in the genus Sarcoptes (Acari: Sarcoptidae) in China. Int J Acarology. 2008;34(2):183-90.

34. Zahler M, Essig A, Gothe R, Rinder H. Molecular analyses suggest monospecificity of the genus Sarcoptes (Acari: Sarcoptidae). Int J Parasitol. 1999;29(5):759-66.

35. Kearse M, Moir R, Wilson A, Stones-Havas S, Cheung M, Sturrock S, Buxton S, Cooper A, Markowitz S, Duran C. Geneious basic: an integrated and extendable desktop software platform for the organization and analysis of sequence data. Bioinformatics. 2012;28(12):1647-9.

36. Edgar RC. MUSCLE: multiple sequence alignment with high accuracy and high throughput. Nucleic Acids Res. 2004;32(5):1792-7.

37. Laslett D, Canback B. ARWEN: a program to detect tRNA genes in metazoan mitochondrial nucleotide sequences. Bioinformatics. 2008;24(2):172-5.

38. Benson DA, Cavanaugh M, Clark K, Karsch-Mizrachi I, Lipman DJ, Ostell J, Sayers EW. GenBank. Nucleic Acids Res. 2013;41(D1):D36-42.

39. Rozas J. DnaSP v5: A software for comprehensive analysis of DNA polymorphism data. Bioinformatics. 2009;25:337-50.

40. Huson DH, Bryant D. Application of phylogenetic networks in evolutionary studies. Mol Biol Evol. 2006;23(2):254-67.

41. Huelsenbeck JP, Ronquist F. MRBAYES: Bayesian inference of phylogenetic trees. Bioinformatics. 2001;17(8):754-5

42. Mofiz E, Seemann T, Bahlo M, Holt D, Currie BJ, Fischer K, Papenfuss AT. Mitochondrial genome sequence of the scabies mite provides insight into the genetic diversity of individual scabies infections. PLoS Negl Trop Dis. 2016;10(2):e0004384

43. Rozas J. DnaSP v5: a software for comprehensive analysis of DNA polymorphism data. Bioinformatics. 2009;25:337-50.

44. Andriantsoanirina V, Ariey F, Izri A, Bernigaud C, Fang F, Charrel R, Foulet F, Botterel F, Guillot J, Chosidow O. Sarcoptes scabiei mites in humans are distributed into three genetically distinct clades. Clin Microbiol Infec. 2015:21(12):1107-14

45. Mounsey KE, McCarthy JS, Walton SF. Scratching the itch: new tools to advance understanding of scabies. Trends Parasitol. 2012;29(1):35-42.

46. Plomley NJB. Friendly Mission. The Tasmanian Journals and Papers of George Augustus Robinson 1829-1834: Queen Victoria Museum and Art Gallery. Launceston: Quintus Publishing; 2008.

47. Powell M. Mosquito Brutality and Exile. Aboriginal Resistance in New South Wales and Van Diemen's Land: Fullers Bookshop Pty Ltd; 2016.

48. Brower A. Rapid morphological radiation and convergence among races of the butterfly Heliconius Erato inferred from patterns of mitochondrial DNA evolution. Proc Natl Acad Sci. 1994;91(14):6491-5.

49. Palopoli MF, Fergus DJ, Minot S, Pei DT, Simison WB, Fernandez-Silva I, Thoemmes MS, Dunn RR, Trautwein M. Global divergence of the human follicle mite Demodex folliculorum: persistent associations between host ancestry and mite lineages. Proc Natl Acad Sci. 2015;112(52):15958-63.

50. Vila $M, B j o ̈ r k l u n d ~ M$. The utility of the neglected mitochondrial control region for evolutionary studies in Lepidoptera (Insecta). J Mol Evol. 2004;58(3):280-90.

51. Arif I, Khan H. Molecular markers for biodiversity analysis of wildlife animals: a brief review. Anim Biodivers Conserv. 2009;32(1):9-17.

52. Skerratt LF, Beveridge I. Human scabies of wombat origin. Aust Vet J. 1999;77(9):607.

53. Arlian LG, Runyan RA, Estes SA. Cross infestivity of Sarcoptes scabiei. J Am Acad Dermatol. 1984:10(6):979-86. 